\title{
Physiological Status of HIV Infected Patients Pre ART
}

\author{
Vinay Malik $^{1 *}$, Tung Vir Singh Arya ${ }^{1}$ and Aman Prakash Garg ${ }^{2}$ \\ ${ }^{1}$ LLRM Medical College and Hospital, Meerut, (UP), India \\ ${ }^{2}$ Shobhit Deemed University, Meerut, (UP), India \\ *Corresponding author
}

\section{A B S T R A C T}

\begin{tabular}{l} 
Ke y w o r d s \\
HIV, Anti- \\
Retroviral therapy, \\
CD4 count, Liver \\
damage, Renal \\
function, \\
Hematological \\
status, IL-6, Sex \\
hormones, \\
Vitamin D \\
\hline $\begin{array}{l}\text { Article Info } \\
\text { Accepted: } \\
10 \text { August } 2020 \\
\text { Available Online: } \\
10 \text { September } 2020\end{array}$ \\
\hline
\end{tabular}

Human Immunodeficiency Virus (HIV) has emerged as one of the most devastating human pandemics with significant morbidity and mortality. A plethora of physiological factors including haematological status, immunological response, renal and liver enzymes, sex hormones, vitamin and mineralogical status play significant role in determining the HIV infection progression and effect of anti-retro viral therapy (ART). The present study aims to investigate the overall physiological status in HIV infected individuals prior to ART. The study was conducted as a quasi-experimental study where HIV infected males and females at Stage I and III of HIV were assessed for their CD4 count, IL-6 content, biochemical, heamatological, immunological, hormonal and Vitamin D functions. Marked variations were noticed in majority of the study variables when compared with the standard defined values. Statistical significant results were obtained in case of total RBCs content and platelet distribution width (PDW) when compared within the disease stages along with increasing trend of few variables with disease progression. It is believed that baseline assessment of physiological parameters prior to treatment initiation will provide greater understanding of disease progression with better treatment outcomes.

\section{Introduction}

HIV infection has emerged as one of the most devastating human pandemics with significant morbidity and mortality. However, with the advent of antiretroviral therapy (ART) the course of this pandemic is remarkably altered therefore enhancing the survival of millions of HIV positive people (Günthard, et al., 2016; Broder, 2010; Bor, et al., 2013, Ndirangu, et al., 2010). It is estimated that more than $70 \%$ of the HIV infected people will be aged more than 50 years by 2030
(Gayle, and Hill, 2001; Weiss,2003). Although antiretroviral therapy has transformed the HIV infection status from non-curable to treatable and manageable, complications associated with the disease course and appearance of associated comorbid conditions still remains a concern for people living with HIV.

A plethora of physiological factors such as liver function analysed through the enzymatic activity, thyroid function, HIV viral load, immunological status, male and female 
gonadal function, hematologic status, and cellular injury play significant role in determining the HIV infection progression and effect of ART (Barroso, Carlson and Meynell, 2003). For instance, haematological abnormalities such as anaemia have been correlated with the progression of HIV infection as well as increased morbidity and mortality of HIV infected patients (Munyazesa, et al., 2012). Similarly, overexpression of IL-6 cytokine has been linked with HIV infection which further contributes to the B-cell activation (Breen, et al., 1990). Low levels of testosterone and dehydroepiandrosterone (DHEA) were reported to be associated with disease progression in people with HIV infection (Wong, et al., 2017). Hypo-vitaminosis D islinked with the exacerbated inflammation and immune response and low peripheral blood CD4+ T-cells in HIV patients resulting in rapid disease progression and short survival time for HIV-infected patients (JiménezSousa, et al., 2018). On the contrary, increased Vitamin D content and Vitamin D Receptor (VDR) expression were linked with the natural resistance to HIV-1 infection.

Considering the importance of multiple physiological variables in the disease progression as well as treatment outcomes, assessment of the status of these parameters before starting the ART may provide great benefits to the clinicians in designing best treatment strategies for improved output. The present study aims to assess the status of different physiological parameters at two different stages of HIV infection (Stage 1 and Stage 3) prior to ART among males and females HIV patients.

\section{Materials and Methods}

The study was conducted as a quasiexperimental study with due approval from Institutional Ethical Committee (XXXXXX). HIV positive patients at different disease stages and were naïve to HAART were duly recruited and registered in the study. Inclusion criteria for participants were HIV seropositive, naïve ART, adults with documented gender and date of birth, no previous history of enrolling in ART clinical studies, and voluntarily ready to give a signed informed consent. The exclusion criteria include pregnant women, nursing mothers, age less than 18 years and non-consenting HIV patients.

Blood collection was done to perform CD4 count, IL-6, biochemical, heamatological, immunological, hormonal and Vitamin D analysis using automated analyzers. Data was analyzed using Graph prism pad software version 8 and mean value plus SEM was calculated for each variable. The statistical output was obtained using One Way ANOVA followed by Posthoc analysis through Tukey's test. Correlation coefficient was determined using Pearson's correlation test. P value less than 0.05 was considered as significant.

\section{Results and Discussion}

\section{CD4 count and IL-6 levels in HIV patients}

A total of 17 male and 14 females' samples were analyzed from Stage 1 whereas 18 male and 13 female samples were studied from Stage 3. In Stage 1 HIV patients, the mean age of males and females were $37.29 \pm 12.31$ and $30.57 \pm 9.64$ years whereas in stage 3 $\mathrm{HIV}$ it was $32.39 \pm 11.48$ and $30.69 \pm 9.99$ years respectively. Figure 1 demonstrated the CD4+ count at Stage 1 and 3. In both the stages an overall decrease in the CD4 count from normal range of 500 - 1500 per cubic $\mathrm{mm}$ was noticed however, no significant differences were obtained in comparison with each other. While in males a decreasing trend in CD4 count was noticed when compared between Stage 1 and 3, interestingly vice a versa was noticed in case of females. 
Alterations in IL-6 levels at Stage 1 and Stage 3 of HIV were represented in Figure 2. In comparison to the reported normal range of IL-6 (5-15 pg/mL), a sharp over expression of IL- 6 content was noticed in both the stages of HIV males and females, however alike CD4 this difference was statistically nonsignificant when compared among each other. Further, while in males an increased IL-6 expression was found both in comparison to females as well as at Stage 3 in comparison to Stage 1, no major changes were observed in case of females.

\section{Hematological Status in HIV patients}

Differences in hematological cells status at Stage 1 and 3 of HIV prior to ART are demonstrated in Table 1. An overall decrease in hemoglobin content was noticed in males and females at both disease stages when compared with the standard normal range. However, there was no significant difference in haemoglobin content among the Stage 1 and 3 males and females.

In case of total Red Blood Cells (RBCs) content, while in males an overall reduced content was noticed at both disease stages when compared with standard normal range (4.7-6.1million cells/L), it was found to be within standard normal range (4.2-5.4 million cells/L) among females. The statistical analysis shows significant decrease in total RBC content between male at stage 3 HIV and females at stage 1 HIV pre-ART $\left({ }^{\# \#} \mathrm{P}<0.01\right)$ and male vs female at stage $3 \mathrm{HIV}$ $\left({ }^{\$} \mathrm{P}<0.01\right)$. Unlike RBCs content, the associated red blood cell indices - mean corpuscular hemoglobin $(\mathrm{MCH})$ and mean corpuscular hemoglobin concentration (MCHC) showed no deviation either in comparison to the standard range or within the study groups. However, another associated red blood index, mean corpuscular volume $(\mathrm{MCV})$ showed an overall marginal decrease from standard normal range of 8096fL/red cell, however no statistical significant changes were recorded within the study groups. Another parameter among the RBC test include Red Cell Distribution Width (RDW) showed no deviations both in case of standard values as well as within study groups.

The standard normal value of Total Leukocyte Count (TLC) is 4000 - 11000 per microliter. While an overall elevated TLC content was found among males at both disease stages, marked reduction was noticed between Stage 1 and Stage 3. In case of females, while in Stage 1 remarkable increase in the TLC content more than the standard range was found, it was decreased at Stage 3. Further, in males much higher TLC was seen in both disease stages when compared with females, however no statistical significance was obtained in any of the cases. Similarly, an acute overall loss of neutrophils, lymphocytes and eosinophils count was noticed at all disease stages when compared with standard values indicating the disease progression. However, it was found to be statistically insignificant when compared between Stage 1 and 3 HIV males and females. On the contrary, platelet count was found to be within standard normal range of $1.5-4.5$ Lakh/ul in all the groups. Although, no statistical significant difference was obtained, a clear reduction in the platelet content with disease progression was noticed both in case of males and females naïve HIV patients. Alike platelet count, similar trend was noticed in Platelet Distribution Width (PDW) with all values lying within standard range of $8.3-25$ fL. Further, a marginal reduction was also noticed with disease progression with significant statistical difference between Stage 1 male and Stage 3 female. While no major deviations were noticed in Mean Platelet Volume (MPV) values both in terms of standard values as well as within the 
disease stage, partial increase in Large Platelet Concentration Ratio (LPCR) content was noticed with disease progression among male and female HIV patients. However, none of the variables showed statistical significant difference. In case of PCT, an overall increase was noticed when compared with standard range of $0.15-0.62 \%$, albeit no major differences between the study groups.

\section{Vitamin D and Sex Hormones Content in HIV naïve patients}

Figure 3 illustrated the status of Vitamin D levels in males and females HIV patients at Stage 1 and Stage 3. Irrespective of the disease status, Vitamin D levels were noticed to be deficient in both males and females when compared with the defined ranges where $<20 \mathrm{ng} / \mathrm{mL}$ is considered to be deficient. Further, no statistical significant differences were seen when compared between gender or disease level.

Level of estrogen and testosterone in female and male HIV patients respectively showed no significant changes when compared between Stage 1 and Stage 3 of HIV disease (Figure 4).

\section{Biochemical parameters in HIV naïve patients}

Table 2 represented the biochemical tests outcomes assessed in HIV naïve patients at Stage 1 and 3. The general renal functioning was assessed through uric acid, urea, protein, albumin and creatinine. No significant deviations were noticed in case of albumin and creatinine both in terms of standard normal range as well as within the studies groups. On the contrary, while protein content was found to be slightly elevated, marked increase in urea and uric acid was noticed when compared with the normal standard range. However, no statistical significant differences were obtained in any of the renal parameters.

The liver functioning among HIV naïve patients were assessed through bilirubin, serum glutamate oxaloacetate transaminase (SGOT), serum glutamic pyruvate transaminase (SGPT) and alkaline phosphatase (ALP) enzymes. While the total bilirubin content was found to be within the normal standard range of $0.1-1.2 \mathrm{mg} / \mathrm{dL}$, marked increase was noticed in direct bilirubin measurement when compared with standard value which is less than $0.3 \mathrm{mg} / \mathrm{dL}$. Marked elevation in SGOT and ALP content was noticed when compared with standard defined values. Further, elevation in these enzymes were observed with disease progression both in males and females in case of ALP whereas in males only in case of SGOT. On the contrary, SGPT levels were found to be within normal standard range of 7-56 units/L serum, except for Stage 3 HIV males where a nominal increase in enzyme activity was found. However, similar to renal functioning assays no statistical significant differences were obtained in any of the liver enzymes activity.

An overall elevated triglycerides level was recorded in HIV naïve patients when compared with the normal value which is less than $150 \mathrm{mg} / \mathrm{dL}$. An increase in their levels was recorded with disease progression, however no statistical significant changes were found. In case of plasma glucose level also no deviations both in terms of normal range as well as within the groups were noticed.

\section{Correlations}

Significant associations between CD4 count, Vitamin D, sex hormones, hematological, and biochemical parameters at different HIV stages in male and female HIV patients were 
also studied using Pearson's correlation. In females who were at Stage 1 of HIV, a single negative correlation between eosinophils and direct bilirubin content $(\mathrm{r}=0.680, \mathrm{p}=$ 0.00744) was obtained.

However, in case of males at Stage 1 of HIV several correlations were obtained. Positive association was obtained between IL-6 and SGOT ( $\mathrm{r}=0.798, \mathrm{p}=0.0001)$, PCT and direct bilirubin $(\mathrm{r}=0.523, \mathrm{p}=0.031)$, SGOT and urea $(\mathrm{r}=0.738, \quad \mathrm{p}=0.0007)$. On the contrary, negative correlation was found between hemoglobin and ALP ( $\mathrm{r}=-0.507, \mathrm{p}=0.037$, eosinophil and direct bilirubin $(\mathrm{r}=-0.502, \mathrm{p}=$ 0.040). Similarly, in females who were at Stage 3 of HIV, negative correlation was obtained between CD4+ count and urea $(r=-$ 0.607, $\mathrm{p}=0.028$ ). On the contrary, positive correlation was found in between eosinophils content and MCV ( $\mathrm{r}=0.785, \mathrm{p}=0.001)$.

In males who were at Stage 3 of HIV, a total of 7 positive correlations were found. The PCT values were positively correlated to direct bilirubin content as well as to the urea with $\mathrm{r}=0.599, \mathrm{p}=0.008$ and $\mathrm{r}=0.687, \mathrm{p}=$ 0.0016 respectively. Direct bilirubin content was positively correlated to Uric acid and urea content with $\mathrm{r}=0.548, \mathrm{p}=0.018$ and $\mathrm{r}=$ $0.602, \mathrm{p}=0.008$ respectively. SGPT was positively correlated with urea and triglycerides with $\mathrm{r}=0.524, \mathrm{p}=0.026$ and $\mathrm{r}=$ $0.631, p=0.005$ respectively. SGOT was positively correlated with urea $(\mathrm{r}=0.770, \mathrm{p}=$ 0.0002). On the other side, a total of 3 negative correlations between total RBCs and SGOT ( $\mathrm{r}=-0.666, \mathrm{p}=0.002)$, SGPT $(\mathrm{r}=-$ $0.679, \mathrm{p}=0.0019)$ and Urea $(\mathrm{r}=-0.735, \mathrm{p}=$ $0.0004)$.

The present study demonstrated the physiological status of HIV naïve males and females at Stage 1 and Stage 3 of HIV. According to the WHO clinical classification, HIV can be categorized into four stages viz.
Stage I (asymptomatic with persistent generalized lymphadenopathy), Stage II (unexplained weight loss, recurrent respiratory tract infections and oral ulceration, herpes zoster, angular cheilitis, popular pruritic eruptions, seborrhoeic dermatitis, and fungal nail infections), Stage 3 (unexplained severe weight loss, chronic diarrhea, persistent fever and oral candidiasis, oral hairy leukoplakia, pulmonary tuberculosis, severe bacterial infections, acute necrotizing ulcerative stomatitis, gingivitis or periodontitis, unexplained anaemia $(<8 \mathrm{~g} / \mathrm{dl})$, neutropaenia $(<0.5 \times 109$ per litre $)$, and/or chronic thrombocytopaenia $(<50 \times 109$ per litre); Stage IV (HIV wasting syndrome; pneumocystis and bacterial pneumonia, herpes infection, oesophageal candidiasis, extrapulmonary tuberculosis and cryptococcosis, Kaposi's sarcoma, cytomegalovirus infection, central nervous system toxoplasmosis, non-tuberculous mycobacterial infection, multifocal leukoencephalopathy, chronic cryptosporidiosis and isosporiasis, mycosis and septicaemia, lymphoma and invasive cervical carcinoma, leishmaniasis, HIVassociated nephropathy, cardiomyopathy, encephalopathy) (WHO, 2006).

In the present study an overall decrease in the CD4 count was noticed in males and females HIV patients at both the stages, however there was no significant difference between them. Role of CD4 cell count as a marker in the assessment of patient's immune status, prognosis of disease progression, life expectancy or mortality as well as ART treatment and its adherence is widely reported (Eholie, et al., 2016; Hogg, et al., 2001; Johnson, et al., 2013; Bock, et al., 2016). In case of ART treatment initiation, two large randomized trials together with recent WHO guidelines revealed that early administration of ART at CD4 cell counts above 500 can effectively reduce HIV transmission, 
morbidity and mortality (Lundgren, et al., 2015; Danel, et al., 2015; Cohen, et al., 2016; World Health Organization, 2015). In the present study, CD4 count ranges from $343-$ 436 thereby indicating the eligibility for treatment initiation. Further, the present results also demonstrated the higher CD4 count in females at least in Stage 3 on one side and faster of CD4 cells in males suggesting enhanced disease progression in males. This is in corroboration with previous published reports where females were reported to have higher mean CD4 cell count than the males (Torpey, et al., 2009; Baveewo, et al., 2011).

Marked over expression of IL-6 content was noticed in both the stages of HIV males and females when compared with standard normal values, however alike CD4 this difference was statistically non-significant when compared among each other. Similar outcomes were reported in previous studies with significant increase in plasma IL-6 levels in HIV seropositive patients in comparison to seronegative individuals (de Medeiros, et al., 2016; Mugwe, et al., 2016). Increased IL-6 expression in HIV naïve patients has been attributed to the increased availability of HIV particles in the pre-HAART stage and is already been associated with morbidity, mortality and development of opportunistic infections (Lafeuillade, et al., 1991; Duprez, et al., 2012; Kuller, et al., 2008; Fuster, et al., 2014).

Another study outcome was an increased IL-6 expression in males when compared with females and within Stage 1 and 3, however no major changes were observed in case of females. This could be justified with another study finding where significant increase in IL6 levels with time was found in HIV naïve patients whose CD4 count decreases more progressively than with relatively stable CD4 count (de Medeiros, et. al., 2016). In our case, we have seen that males have much higher rate of CD4 count decrease and IL-6 level increase than females further suggesting higher disease progression rate.

Cytopenias are widely evident in HIV condition with further disease progression. In the present study, aneamic conditions as evident by reduced haemoglobin and RBCs content was found in HIV naïve males and females at both disease stages when compared with the standard normal range. This is in accordance with previous reports where anemia is reported to be one of the most prevalent cytopenias associated with HIV (Igbeneghu, et al., 2014).

Further, lack of gender difference was observed in case of haemoglobin loss and microcytic anemia suggesting iron deficiency as not the primary cause of anemia in the present study population. However, converse to the majority of previous studies, the present study found more RBCs loss in males when compared to females, further justifying the association between prevalence of anemia with the degree of immunosuppression (Igbeneghu, et al., 2014; Bleyere, et al., 2013; Sullivan, et al., 1998; Akinbami, et al., 2010; Masaisa et al., 2011).

In case of White Blood Cells (WBCs) status, although an overall marked elevation in TLC content was found at both disease stages, it was found to be reduced with disease progression. This is in accordance with previous published reports where high WBCs content was revealed in HIV naïve patients (Ratnam, et al., 2017). Further, an overall decrease in neutrophils (neutropenia), lymphocytes (lymphopenia), and eosinophils were noticed in the study which is in concomitance with previous studies (Fekene, et al., 2018; Kusfa, et al., 2017; Katemba, et al., 2018). 
Table.1 Comparative Analysis of Red Blood Cell and White Blood Cells Indices among Stage 1 and 3 male and female patients prior to ART

\begin{tabular}{|c|c|c|c|c|}
\hline \multirow[t]{2}{*}{ Hematological Parameters } & \multicolumn{2}{|c|}{ Stage 1} & \multicolumn{2}{|c|}{ Stage 3} \\
\hline & Male & Female & Male & Female \\
\hline Haemoglobin $(\mathrm{g} / \mathrm{dL})$ & $11.42 \pm 2.31$ & $10.64 \pm 1.73$ & $10.63 \pm 1.78$ & $11.75 \pm 2.50$ \\
\hline Total RBC count (millions/L) & $4.24 \pm 0.67$ & $4.71 \pm 0.5$ & $3.89 \pm 0.64^{\# \#}$ & $4.70 \pm 0.72^{\$ \$}$ \\
\hline MCV (fL/red cell) & $74.86 \pm 8.49$ & $74.16 \pm 5.22$ & $76.15 \pm 7.29$ & $76.23 \pm 8.89$ \\
\hline MCH (pg/red cell) & $26.87 \pm 2.80$ & $27.26 \pm 2.96$ & $27.08 \pm 2.95$ & $27.68 \pm 2.92$ \\
\hline MCHC (g/dL) & $35.01 \pm 1.99$ & $34.08 \pm 0.75$ & $34.04 \pm 1.16$ & $34.83 \pm 1.24$ \\
\hline RDW (\%) & $13.65 \pm 2.10$ & $12.85 \pm 1.24$ & $14.03 \pm 1.63$ & $13.27 \pm 1.33$ \\
\hline RDWA & $63.24 \pm 30$ & $46.90 \pm 15.01$ & $58.84 \pm 25.51$ & $59.14 \pm 30.71$ \\
\hline TLC $($ per $\mu \mathbf{L})$ & $\begin{array}{c}25947.06 \pm \\
28304.84\end{array}$ & $\begin{array}{c}11157.14 \pm \\
19289.08\end{array}$ & $\begin{array}{c}15500 \pm \\
23866.66\end{array}$ & $\begin{array}{c}6738.46 \pm \\
1832.37\end{array}$ \\
\hline Neutrophils $\left(\mathrm{mm}^{3}\right)$ & $60.64 \pm 9.17$ & $60.36 \pm 10.83$ & $62.32 \pm 6.92$ & $65.69 \pm 11.35$ \\
\hline Lymphocytes (per $\mu \mathrm{L})$ & $32.66 \pm 7.69$ & $33.14 \pm 11.09$ & $33.54 \pm 8.07$ & $33.62 \pm 8.09$ \\
\hline Eosinophils (per $\mu \mathrm{L})$ & $3.02 \pm 2.16$ & $2.64 \pm 0.63$ & $3.24 \pm 2.93$ & $3.15 \pm 1.72$ \\
\hline Platelet Count (Lacs per $\mu \mathrm{L})$ & $4.59 \pm 12.00$ & $2.12 \pm 1.21$ & $1.95 \pm 1.12$ & $1.96 \pm 0.78$ \\
\hline PDW (fL) & $13.46 \pm 2.35$ & $12.04 \pm 1.64$ & $12.77 \pm 2.12$ & $11.40 \pm 0.74^{*}$ \\
\hline MPV (fL) & $10.35 \pm 1.23$ & $10.91 \pm 0.81$ & $10.41 \pm 1.19$ & $10.36 \pm 1.27$ \\
\hline LPCR & $10.27 \pm 6.13$ & $10.58 \pm 6.88$ & $12.42 \pm 7.20$ & $13.22 \pm 8.68$ \\
\hline PCT (\%) & $24.69 \pm 10.48$ & $29.10 \pm 9.95$ & $25.13 \pm 9.22$ & $25.07 \pm 11.73$ \\
\hline
\end{tabular}

Data represented as Mean \pm SEM. Statistical analysis was performed using One Way ANOVA. $* \mathrm{P}<0.05$ male at stage $1 \mathrm{HIV}$ and females at stage $3 \mathrm{HIV} ;{ }^{\# \#} \mathrm{P}<0.01$ male at stage $3 \mathrm{HIV}$ and females at stage $1 \mathrm{HIV} ;{ }^{\$ \$} \mathrm{P}<0.01$ male vs female at stage 3 HIV

Table.2 Comparative Analysis of the biochemical tests data perform to study the renal function among Stage 1 and 3 male and female patients prior to ART

\begin{tabular}{|c|c|c|c|c|}
\hline \multirow{2}{*}{ Biochemical Parameters } & \multicolumn{2}{|c|}{ Stage 1 } & \multicolumn{2}{c|}{ Stage 3 } \\
\cline { 2 - 5 } & Male & Female & Male & Female \\
\hline Proteins (g/dL) & $8.76 \pm 1$ & $8.61 \pm 0.93$ & $8.46 \pm 1.13$ & $8.62 \pm 0.94$ \\
\hline Albumin (g/dL) & $3.45 \pm 0.64$ & $3.42 \pm 0.65$ & $3.46 \pm 0.49$ & $3.79 \pm 0.52$ \\
\hline Uric acid (mg/dL) & $8.36 \pm 1.73$ & $7.30 \pm 1.53$ & $7.67 \pm 2.05$ & $9.56 \pm 7.63$ \\
\hline Urea (mg/dL) & $32.89 \pm 14.35$ & $33 \pm 5.76$ & $40.73 \pm 16.28$ & $31.55 \pm 9.32$ \\
\hline Creatinine (mg/dL) & $1.1 \pm 0.26$ & $0.96 \pm 0.41$ & $0.99 \pm 0.37$ & $0.79 \pm 0.24$ \\
\hline Bilirubin, Total (mg/dL) & $0.64 \pm 0.28$ & $0.74 \pm 0.35$ & $0.97 \pm 0.54$ & $0.67 \pm 0.33$ \\
\hline Bilirubin, Direct (mg/dL) & $0.49 \pm 0.17$ & $0.47 \pm 0.17$ & $0.53 \pm 0.21$ & $0.44 \pm 0.21$ \\
\hline Bilirubin, Indirect & $0.39 \pm 0.05$ & $0.37 \pm 0.11$ & $0.38 \pm 0.07$ & $0.39 \pm 0.11$ \\
\hline SGOT (units/L serum) & $47.35 \pm 29.27$ & $46.64 \pm 23.83$ & $62.03 \pm 36.40$ & $44.08 \pm 18.18$ \\
\hline SGPT(units/L serum) & $46.62 \pm 29.11$ & $36.14 \pm 14.97$ & $58.03 \pm 36.27$ & $49.71 \pm 22.45$ \\
\hline Alkaline Phosphatase (IU/L) & $451.41 \pm 320.76$ & $296.54 \pm 263.80$ & $584.54 \pm 407.23$ & $325.1 \pm 151.85$ \\
\hline Triglycerides (mg/dL) & $223.91 \pm 88.92$ & $222.99 \pm 99.55$ & $261.22 \pm 120.66$ & $229.88 \pm 102.3$ \\
\hline Plasma Glucose Random & $99.85 \pm 16.51$ & $96.49 \pm 37.32$ & $88.49 \pm 14.42$ & $98.55 \pm 31.28$ \\
\hline (mg/dL) & & & & \\
\hline
\end{tabular}

Data represented as Mean \pm SEM. Statistical analysis was performed using One Way ANOVA. No statistical differences was obtained 
Fig.1 Differences in CD4 count at Stage 1 and 3 in males and females

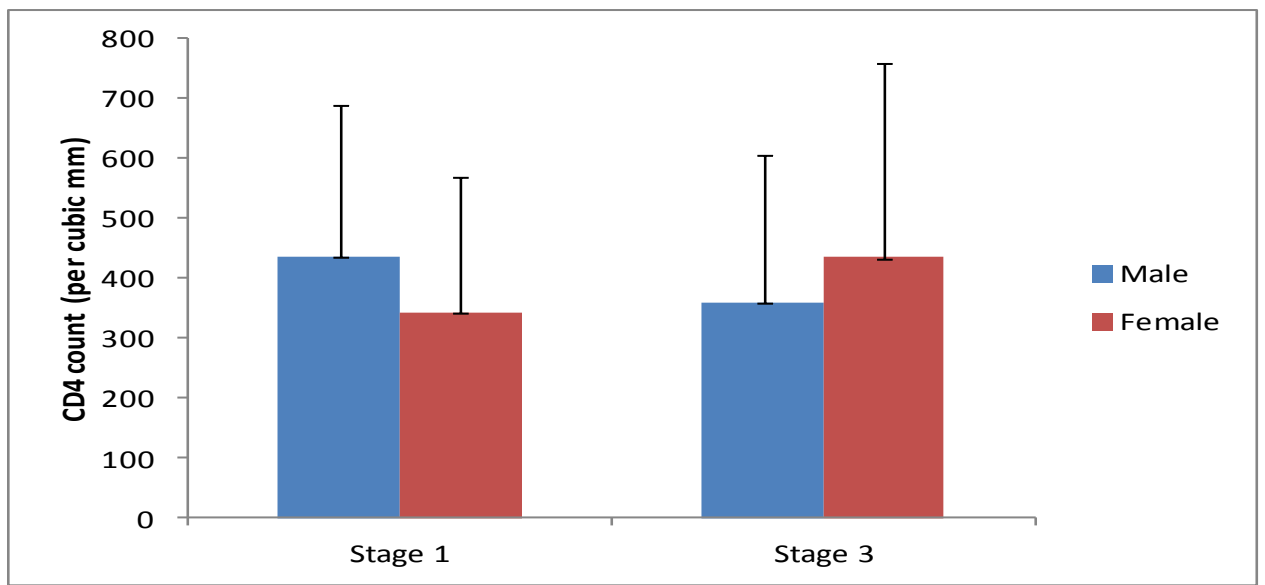

Data represented as Mean \pm SEM. No statistical differences was obtained

Fig.2 Alterations in IL-6 levels at Stage 1 and 3 HIV males and females

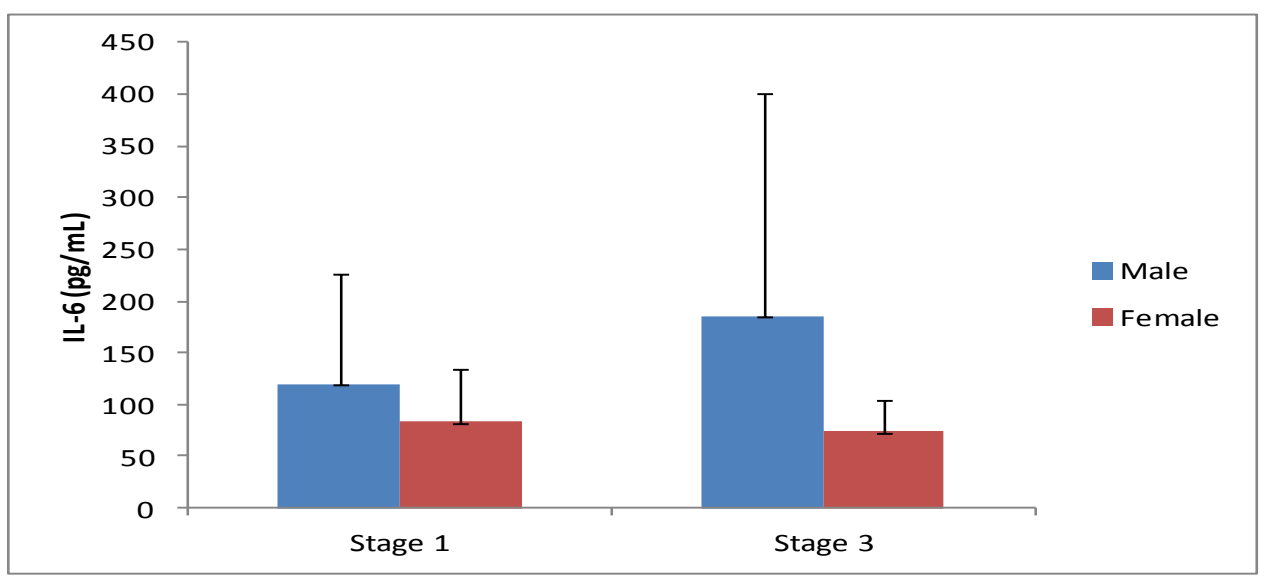

Data represented as Mean \pm SEM. Statistical analysis was performed using One Way ANOVA. No statistical differences was obtained

Fig.3 Status of Vitamin D levels in Stage 1 and 3 HIV males and females

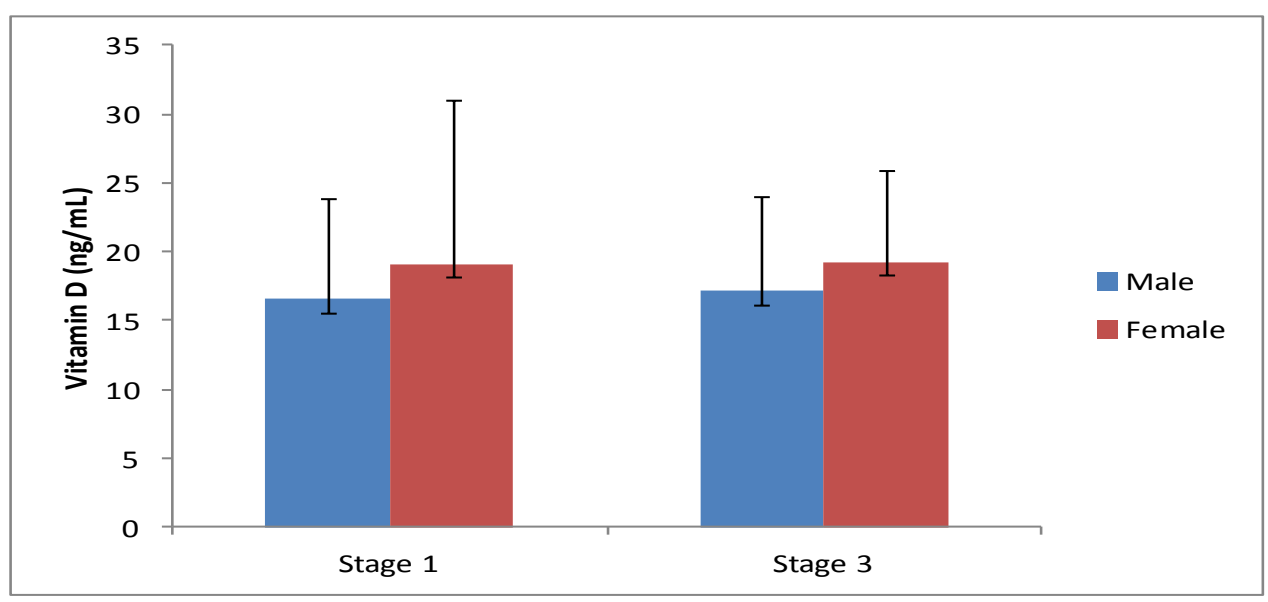


Data represented as Mean \pm SEM. No statistical differences was obtained

Fig.4 Level of estrogen and testosterone in females and males HIV patients respectively at Stage 1 and 3

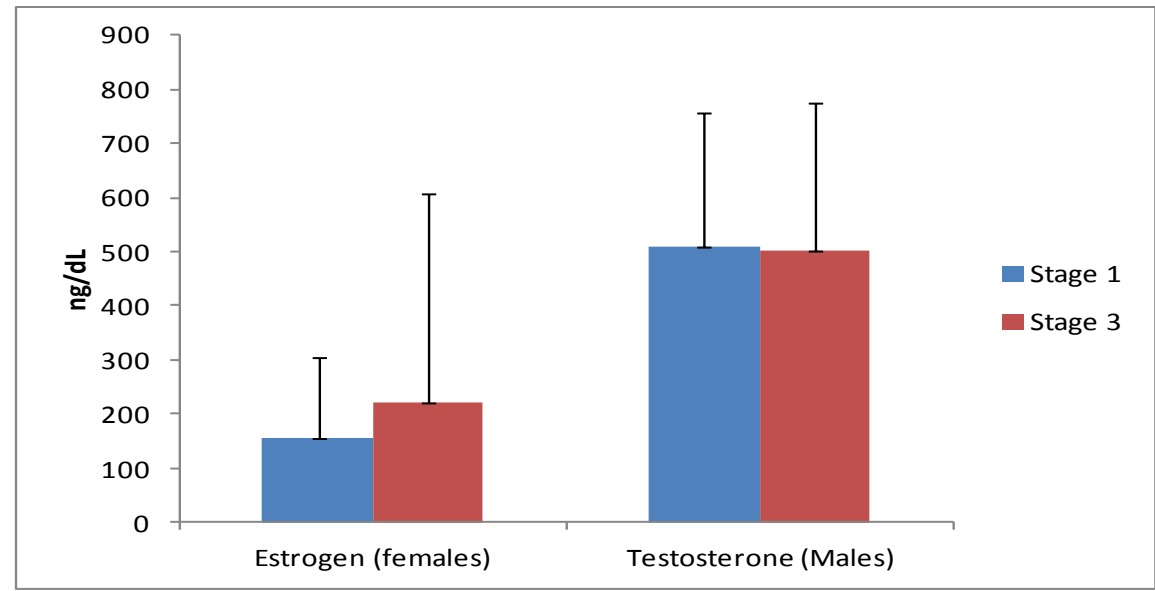

Data represented as Mean \pm SEM. No statistical differences was obtained Biochemical parameters in HIV naïve patients

Neutropenia is majorly attributed to the HIV induced suppression of bone marrow and synthesis of antigranulocyte antibodies leading to abnormal granulopoiesis and decreased production of granulocyte colony stimulating factor respectively (Kimura, et al., 1990; Mauss, et al., 1997). In converse to the previous studies, the present study reported normal platelet counts in all disease stages (Igbeneghu, et al., 2014; Munyazesa, et al., 2012).

This finding is also in consonance with the observation that the prevalence of thrombocytopenia is independent of the degree of immunosuppression and clinical stage of HIV (Adewuyi, et al., 1999; Akinbami, et al., 2010). The present study also found an independent positive correlation between eosinophils content and MCV suggesting that HIV stage may significantly contribute in hematological abnormalities before treatment.

Irrespective of the disease status, the present study outcomes demonstrated hypo vitaminosis $\mathrm{D}$ in both males and females when compared with the standard range, similar to the previous published studies. These reports revealed insufficient to deficient vitamin D levels in HIV-1 infected individuals (Barbosa, Costa, Pinto, 2014; Thacher, Clarke, 2011; Holick, et al., 2011). Vitamin D deficiency in HIV infected individuals were correlated with several comorbidities and increased predisposition to several disorders such as osteopenia, osteoporosis, cardiovascular disease (CVD), and diabetes mellitus due to its immunomodulatory, anti-inflammatory, and antimicrobial properties (Lake, Adams, 2011; Prietl, et al., 2013; Radovic, et al., 2012; Bander and Parczewski, 2012; Lai, et al., 2013).

No significant changes in estrogen and testosterone levels among female and male HIV patients respectively were found in any of the disease stage, converse to the previous published reports where both sex hormones were reported to be compromised with HIV infection (Kumari, et al., 2018; Nkiruka, et al., 2017; Aggarwal, et al., 2018). These deviations may perhaps due to the disease status as these studies showed linear relationship between compromised sex 
hormones and disease status. Further, the presence of normal estrogen levels in females can in part explain the low disease status as estrogen has been reported to be a potent inhibitor of HIV transcription in latency models and primary cells (Szotek et al., 2013; Scully, 2018).

Research outcomes of the present study demonstrated no significant deviations in albumin and creatinine content on one side whereas marked increase in urea and uric acid was noticed on the other side when compared with standard reference range. Further, while in males an increase in urea content was found with disease stage, similar trend was noticed in case of uric acid among females. In-addition, marginal elevation in protein content was also noticed in all groups.

All these outcomes are in accordance with recent published reports suggesting renal insufficiency (Adeleke and Emokpae, 2019; Devi, et al., 2019). Hyperuricemia has been extensively reported in HIV infection and indicates either renal insufficiency or body's adaptive mechanism to combat HIVassociated excessive free radicals generation (Izzedine and Deray, 2007). Similarly, elevated urea content suggest compromised filtration process of kidney and pre-renal uremia attributed either to high protein intake or hyper catabolic states including muscle wasting (Eneyew, et al., 2016; Bello, Onunu and Erah, 2014). The present study further noticed few independent associations between urea and other disease covariates at Stage 3. While in females a negative correlation was obtained between CD4+ count and urea, in males urea was positively and negatively correlated with PCT and total RBCs respectively.

The liver functioning outcomes of HIV naïve patients revealed marked increase in direct bilirubin, SGOT and ALP content at all disease stages. Further, while ALP was found to be elevated with disease progression both in males and females HIV patients, this trend was noticed among males in case of SGOT. On the contrary, total bilirubin content and SGPT was found to be within the normal standard range in HIV-infected males and females. These results are in concomitance with previous published reports where HIV serostatus was significantly associated with liver damage markers (Dusingize, et al., 2015; Agbecha and Ikyernum, 2018; Ebot, et al., 2015). Multiple direct or indirect mechanisms were reported to be involved in HIV associated liver damage. Direct mechanisms include HIV infection of hepatic cells such as Kupffer cells, sinusoidal cells, hepatocytes, hepatic stellate cells via CD4 independent mechanisms mainly receptor-mediated endocytosis, expression of alternative coreceptors, or hepatic cells apoptosis. Indirect mechanisms involve HIV induced increased permeability of bacterial endotoxins such as lipopolysaccharide in GI tract which in turn targets liver Kupffer cells. Together, this results in chronic immune activation as well as synthesis of pro-inflammatory cytokines by Kupffer cells with consequent liver disease progression as also indicated in this study where ALP and SGOT was increased with disease stage (review by Crane, et. al., 2012). Further, liver damage due to indirect mechanisms were reflected by several correlations obtained in this study such as increased direct bilirubin content with decreased eosinophils amongst HIV infected males and females at Stage I, positive association between increased direct bilirubin content with PCT in HIV infected males at Stage I and III, and linear positive relationship between IL-6 and SGOT level in HIV infected males at Stage I. Further, the present study also observed an independent association between anaemia and high urea content with liver damage markers in males at both disease stages which may also be perhaps driven by an increase in circulating 
inflammatory factors.

An overall increase in triglycerides level was noticed among HIV naïve patients in this study when compared with the standard normal range with an increase with disease progression. Lipid alterations including elevated serum triglycerides are implicated in literature among HIV naïve patients which potentially gets increased with disease progression.

This may be attributed to several factors such as altered cytokine levels and steroid hormones, decreased lipid clearance, and increased hepatic synthesis of VLDL (Grunfeld, et al., 1989; Nguemaïm, et al., 2010; Grunfeld, et al., 1991; Grunfeld and Feingold, 1992). The relationship between liver damage and increased triglycerides content was found in this study where SGOT was positively correlated with triglycerides. Further, the present study found normal plasma glucose level in all study participants.

The major study limitations was the absence of healthy controls as distinct changes were found in most of the study variables when compared with standard defined range, however these cannot be proved statistically. Another study limitation is the small sample size which is not sufficient to reflect the complete scenario of the HIV population. Nevertheless, the study provides a glimpse of the overall physiological status of HIV patients at two disease stages and strongly recommends future work in larger HIV infected population in our country.

Conflict of Interest: The authors have not reported any Conflict of Interest.

\section{References}

Adeleke, T.D., and Emokpae, M.A. (2019). Assessment of Some Biomarkers of Renal Function and Myoglobin Level in Human Immunodeficiency Virus-1 Infected Subjects. Journal of Medical Laboratory Science, 29 (1), 61-72.

Adewuyi, J.O., Coutts, A.M., Latif, A.S., et al., (1999). Haematologic features of the human immunodeficiency virus (HIV) infection in adult Zimbabweans. Cent Afr J Med, 45, 26-30.

Agbecha, A., Ikyernum, J. A. (2018). Impact of HIV-Infection on Serum Liver Enzymes: A Comparative Study among Anti-retroviral Therapy (ART) Naïve Patients, ART Follow-Up Patients, and HIV Sero-negative Controls. International Journal of Healthcare and Medical Sciences, 4(12), 196-200.

Aggarwal, J., Taneja, R.S., Gupta, P.K., et al., (2018). Sex hormone Profile in Human Immunodeficiency Virus-Infected Men and It's Correlation with CD4 Cell Counts Indian. $J$ Endocrinol Metab, 22(3), 328-334.

Akinbami, A., Oshinaike, O., Adeyemo, T., et al., (2010). Hematologic abnormalities in treatment- Naïve HIV patients infectious diseases. Research and Treatment, 3, 45-9.

Bander, D., Parczewski, M.(2012). Osteoporosis and vitamin D deficiency in HIV infected

Barbosa, N., Costa, L., Pinto, M. (2014). Vitamin D and HIV Infection: a systematic review. Immunod Disord, 3,1. doi: 10.4172/2324-853X.1000107.

Barroso, J., Carlson, J.R., Meynell, J. (2003). Physiological and psychological markers associated with HIV-related fatigue. Clin Nurs Res, 12, 49-68.

Baveewo, S., Ssali, F., Karamagi, C., et al., (2011). Validation of World Health Organisation HIV/AIDS Clinical Staging in Predicting Initiation of Antiretroviral Therapy and Clinical Predictors of Low CD4 Cell Count in Uganda. PLoS ONE, 6(5), e19089. doi:10.1371/journal.pone.0019089. 
Bello, S., Onunu, A., Erah, P. (2014). LongTerm Effect of HAART on Biochemical Profiles of HIV/AIDS Patients in a Tertiary Health Facility in Benin City, Nigeria. Tropical $J$ Pharmaceut Res, 13(11), 1941-1946.

Bleyere, M.N., Koukou, L.K., Konan, A.B., et al., (2013). Haematological status and progression of HIV infection in Abidjan (Côte D'ivoire). Int J Med Res Health Sci, 1, 18-30.

Bock, P., James, A., Nikuze, A., et al., (2016).Baseline CD4 count and adherence to antiretroviral therapy: a systematic review and meta-analysis. $J$ Acquir Immune Defic Syndr, 73, 514 521.

Bor, J., Herbst, A.J., Newell, M.L., et al., (2013). Increases in adult life expectancy in rural South Africa: valuing the scale-up of HIV treatment. Science, 339(6122), 961-5.

Breen, E.C., Rezai, A.R., Nakajima, K., et al., (1990). Infection with HIV is associated with elevated IL-6 levels and production. J Immunol, 144(2), 480-484.

Broder, S.(2010). The development of antiretroviral therapy and its impact on the HIV-1/AIDS pandemic. Antivir Res, 85(1), 1-18.

Cohen, M.S., Chen, Y.Q., McCauley, M., et al., (2016). Antiretroviral therapy for the prevention of HIV-1 transmission. N Engl J Med, 375, 830-839.

Crane, M., Iser, D., Lewin, S.R. (2012).Human immunodeficiency virus infection and the liver. World $J$ Hepatol, 4(3), 91-98.

Devi, M.S., Gurtoo, A., Singh, R., Tiwari, S. (2019). Renal Profile in HIV Seropositive Patients on Highly Active Anti Retroviral Therapy (Haart) Compared with Haart-Naïve Patients. International Journal of Science and Research (IJSR), 8 (11).
Duprez, D.A., Neuhaus, J., Kuller, L.H., Tracy, R., Belloso, W. and De Wit, S, (2012). Inflammation, coagulation and cardiovascular disease in HIV-infected individuals. PLoS One, 7,e44454.

Dusingize, J.C., Hoover,D.R.,Qiuhu Shi, Q., et al., (2015). Association of Abnormal Liver Function Parameters with HIV Serostatus and CD4 Count in Antiretroviral-Naive Rwandan Women. Aids research and human retroviruses, $31,7$.

Eholie, S.P., Badje, A., Kouame, G.M., et al., (2016). Antiretroviral treatment regardless of CD4 count: the universal answer to a contextual question. AIDS Res Ther, 13, 27.

Eneyew, K., Seifu, D., Amogne, W., Menon, M. (2016). Assessment of Renal Function among HIV-Infected Patients on Combination Antiretroviral Therapy at Tikur Anbessa Specialized Hospital, Addis Ababa, Ethiopia. Technology and Investment. Scientific Res, 7, 107122.

Fekene,T.E.,Juhar, Mengesha,C.H.,Worku,D.K.(2018). Prevalence of cytopenias in both HAART and HAART naïve HIV infected adult patients in Ethiopia: a cross sectional study. BMC Hematology, 18, 8.

Fuster, D., Cheng, D.M., Quinn, E.K., Armah, K.A., Saitz, R. and Freiberg, M.S, (2014). Inflammatory cytokines and mortality in a cohort of HIV-infected adults with alcohol problems, AIDS, 28, 1059-1064.

Gayle, H. D., and Hill, G. L. (2001).Global impact of human immunodeficiency virus and AIDS. Clinical microbiology reviews, $14(2)$, 327-335. doi:10.1128/CMR.14.2.327-335.2001.

Grunfeld, C., Feingold, K.R. (1992). The role of the cytokines, interferon alpha and tumor necrosis factor in the 
hypertriglyceridemia and wasting of AIDS. J Nutr, 122(3), 749-53.

Grunfeld, C., Kotler, D.P., Hamadeh, R., et al., (1989). Hypertriglyceridemia in the acquired immunodeficiency syndrome. Am J Med, 86, 27-31.

Grunfeld, C., Kotler, D.P., Shigenaga, J.K., et al., (1991). Circulating interferon-alpha levels and hypertriglyceridemia in the acquired immunodeficiency syndrome. Am J Med, 90, 154-62.

Günthard, H.F., Saag, M.S., Benson, C.A., et al., (2016). Antiretroviral Drugs for Treatment and Prevention of HIV Infection in Adults: 2016 Recommendations of the International Antiviral Society-USA Panel. JAMA, 316(2), 191-210. doi:10.1001/jama.2016.8900.

Hogg, R.S., Yip, B., Chan, K.J., et .al. (2001).Rates of disease progression by baseline CD4 cell count and viral load after initiating triple-drug therapy. JAMA, 286,2568-2577.

Holick, M.F., Binkley, N.C., Bischoff-Ferrari, H.A., et al., (2011). Evaluation, treatment, and prevention of vitamin D deficiency: an endocrine society clinical practice guideline. $J$ Clin Endocrinol Metab, 96, 1911-30. doi: 10.1210/jc.2011-0385.

Igbeneghu, C., Odaibo, G.N., Olaleye, D.O., Odaibo, A.B. (2014). Asymptomatic HIV infection and its impact on some haematological parameters in Iwo community, Southwestern Nigeria. World J Med Sci, 10, 375-8.

INSIGHT START Study Group. Lundgren, J.D., Babiker, A.G., Gordin, F., et .al.(2015). Initiation of antiretroviral therapy in early asymptomatic HIV infection. N Engl J Med, 373,795-807.

Izzedine, H., Deray, G. (2007).The Nephrologist in the HAART era. Acquired Immune Def Syndr, 21(4), 409-421.
Jiménez-Sousa, M. Á., Martínez, I., Medrano, L. M., et al., (2018). Vitamin D in Human Immunodeficiency Virus Infection: Influence on Immunity and Disease. Frontiers in immunology, 9, 458. doi:10.3389/fimmu.2018.00458.

Johnson, L.F., Mossong, J., Dorrington, R.E., et al., (2013). Life expectancies of South African adults starting antiretroviral treatment: collaborative analysis of cohort studies. PLoSMed, 10:e1001418.

Katemba, C., Muzoora, C., Muwanguzi, E., et al., (2018). Hematological abnormalities in HIV-antiretroviral therapy naïve clients as seen at an immune suppression syndrome clinic at Mbarara Regional Referral Hospital, southwestern Uganda. Journal of Blood Medicine, 9, 105-110.

Kimura, S., Matsuda, J., Ikematsu, S., et al., (1990).Efficacy of recombinant human granulocyte colony stimulating factor on neutropenia in patients with AIDS.AIDS, 4, 1251-5.

Kuller, L.H., Tracy, R., Belloso, W., De Wit, S., Drummond, F. and Lane, H.C. (2008). Inflammatory and coagulation biomarkers and mortality in patients with HIV infection. PLoS Med, 5, e203.

Kumari, A., Dalal,D.,Kumar,R.,et al., (2018). Should Clinician Suspect Hormonal Disturbances at Early Stages of HIV Infection in Males?- A North Indian Study. International Journal of Research and Review, 1 (5), 4.

Kusfa, I.U., Abubakar, A.A., Muktar, H.M., et al., (2017). Comparative analysis of some hematological and immunological parameters of HIVpositive patients at a tertiary HIV treatment center in Zaria, Nigeria. SubSaharan Afr J Med,4,15-9.

Lafeuillade, A., Poizot-Martin, I., Quillichini, R., et al., (1991). Increased interleukin6 production is associated with disease 
progression in HIV infection. AIDS, 1139. PMID: 1930778.

Lai, S., Fishman, E.K., Gerstenblith, G., et al., (2013).Vitamin D deficiency is associated with coronary artery calcification in cardiovascularly asymptomatic African Americans with HIV infection. Vasc Health Risk Manag, $\quad$ 9, 493-500.doi: 10.2147/VHRM.S48388.

Lake, J.E., Adams, J.S. (2011). Vitamin D in HIV-infected patients. Curr HIV/AIDS Rep, 8,133-41.doi: 10.1007/s11904011-0082-8.

Masaisa, F., Gahutu, J.B., Mukiibi, J., et al., (2011). Anemia in human immunodeficiency virus-infected and uninfected women in Rwanda. Am $J$ Trop Med Hyg, 4, 456-60.

Mauss, S., Steinmetz, H.T., Willers, R., et al., (1997). Induction of granulocyte colony stimulating factor by acute febrile infection but not by neutropenia in HIV seropositive individuals. $J$ Acquir Immune DeficSyndr Hum Retrovirol, 14, 430-4.

Medeiros, R.M., Valverde-Villegas, J.M., Junqueira, D.M., et al., (2016). Rapid and Slow Progressors Show Increased IL-6 and IL-10 Levels in the Pre-AIDS Stage of HIV Infection. PLOS ONE, 11(5),e0156163. doi:10.1371/journal.pone.0156163

Mugwe, J.N., Gicheru, M.M. and Mwatha, J. (2016). Plasma Cytokine Profiles as Predictive Biomarkers of HIV and Aids Progression among HIV Patients Attending Nakuru Provincial General Hospital, Kenya. American Journal of Medical and Biological Research, 4(2), 20-25. doi: 10.12691/ajmbr-4-2-2.

Munyazesa, E., Emile, I., Mutimura, E., et al., (2012). BMJ Open, 2:e01600. doi:10.1136/bmjopen-2012-001600.

Munyazesa, E., Emile, I., Mutimura, E, et al., (2012). Assessment of haematological parameters in HIV-infected and uninfected Rwandan women: a crosssectional study. BMJ Open, 2: pii. e001600. doi: 10.1136/bmjopen-2012001600 .

Ndirangu, J., Newell, M.L., Tanser, F., et al., (2010). Decline in early life mortality in a high HIV prevalence rural area of South Africa: evidence of HIV prevention or treatment impact? AIDS , 24(4), 593.

Nguemaïm, N.F., Mbuagbaw, J., Nkoa, T., et al., (2010). Serum lipid profile in highly active antiretroviral therapynaïve HIV-infected patients in Cameroon: a case-control study. HIV Med, 11, 353-9.

Nkiruka, R.U., Solomon, N.U., Charles, C.O., et al., (2017). Prognostic Value of Serum Estrogen, Cortisol, Calcium and Alkaline Phosphatase Activity in Pre and Postmenopausal HIV Women at Nnewi, Nigeria. International Journal of TROPICAL DISEASE and Health, 21(4), 1-11.

Patients: genetic and classical factors compared to the HIV-associated ones review. HIV AIDS Rev, 11, 1-4. doi: 10.1016/j.hivar.2011.11.001.

Prietl, B., Treiber, G., Pieber, T.R., Amrein, K. (2013). Vitamin D and immune function. Nutrients, 5, 2502-21. doi: 10.3390/nu5072502.

Radovic, J., Markovic, D., Velickov, A., et al., (2012). Vitamin D immunomodulatory effect. Acta Med Med, 51:58-64.doi: 10.5633/amm.2012.0409s

Ratnam, M.V.R., Nayyar, A.S., Prasad, K., Sashikiran, S.V.N., Gupta, U., DebasisSahu, D. (2017). CD4 cell counts, complete blood picture and lipid profile in HIV infected and AIDS patients in a specific populace from South India. The Egyptian Journal of Internal Medicine, 29,151-163. 
Scully, E.P. (2018). Sex Differences in HIV Infection. Current HIV/AIDS Reports, 15,136-146.

Sullivan, P.S., Hanson, D.L., Chu, S.Y., et al., (1998). Epidemiology of Anemia in human immunodeficiency virus (HIV)infected persons: results from the multistate adult and adolescent Spectrum of HIV disease surveillance project. Blood, 91, 301-8.

Szotek, E.L., Narasipura, S.D., Al-Harthi, L. (2013). 17beta-estradiol inhibits HIV-1 by inducing a complex formation between beta-catenin and estrogen receptor alpha on the HIV promoter to suppress HIV transcription. Virology, 443(2): 375-83. https://doi.org/10. 1016/j.virol.2013.05.027.

TEMPRANO ANRS 12136 Study Group.Danel, C, Moh, R., Gabillard, D., et al., (2015). A trial of early antiretrovirals and isoniazid preventive therapy in Africa. $N$ Engl $\mathrm{J}$ Med, $373,808-822$.

Thacher, T.D., Clarke, B.L. (2011).Vitamin D insufficiency. Mayo Clin Proc, 86, 50 60. doi: 10.4016/26528.01.

Torpey, K., Lartey, M., Amenyah, R., et al., (2009). Initiating antiretroviral treatment in a resource-constrained setting: does clinical staging effectively identify patients in need? International journal of STD \&AIDS, 20(6), 395-
398.

Walter, O., Ebot, E.A., Achidi, H.F., et al., (2015). Liver function tests of HIV/AIDS patients at the nylon district hospital, Douala, Cameroon International Journal of Research in Medical Sciences, 3(10), 2549-2552.

Weiss R. A. (2003). HIV and AIDS in relation to other pandemics. Among the viruses plaguing humans, HIV is a recent acquisition. Its outstanding success as an infection poses immense scientific challenges to human health and raises the question "What comes nest?". EMBO reports, 4 (1), S10-S14. doi:10.1038/sj.embor.embor857

Who case definitions of HIV for surveillance and revised clinical staging and immunological classification of HIVrelated disease in adults and children. WHO, 2006.

Wong, N., Levy, M., and Stephenson, I. (2017). Hypogonadism in the HIVInfected Man. Current treatment options in infectious diseases, 9(1), 104-116. doi:10.1007/s40506-0170110-3.

World Health Organization.(2015). Guideline on when to start antiretroviral therapy and on preexposure prophylaxis for HIV. Geneva: WHO.

\section{How to cite this article:}

Vinay Malik, Tung Vir Singh Arya and Aman Prakash Garg. 2020. Physiological Status of HIV Infected Patients Pre ART. Int.J.Curr.Microbiol.App.Sci. 9(09): 1124-1138. doi: https://doi.org/10.20546/ijcmas.2020.909.140 